\title{
Progress on the Microscopic Spectrum of the Dirac Operator for QCD with Wilson Fermions
}

\section{K. Splittorff}

Discovery Center, The Niels Bohr Institute

University of Copenhagen,

Blegdamsvej 17, DK-2100 Copenhagen, Denmark

E-mail: splitenbi.dk

\section{J.J.M. Verbaarschot}

Stony Brook University

Department of Physics and Astronomy

Stony Brook, NY 11794-3800, USA

E-mail: jacobus.verbaarschot@stonybrook.edu

\begin{abstract}
Starting from the chiral Lagrangian for Wilson fermions at nonzero lattice spacing we have obtained compact expressions for all spectral correlation functions of the Hermitian Wilson Dirac operator in the $\varepsilon$-domain of QCD with dynamical quarks. We have also obtained the distribution of the chiralities over the real eigenvalues of the Wilson Dirac operator for any number of flavors. All results have been derived for a fixed index of the Dirac operator. An important effect of dynamical quarks is that they completely suppress the inverse square root singularity in the spectral density of the Hermitian Wilson Dirac operator. The analytical results are given in terms of an integral over a diffusion kernel for which the square of the lattice spacing plays the role of time. This approach greatly simplifies the expressions which we here reduce to the evaluation of two-dimensional integrals.
\end{abstract}

The XXIX International Symposium on Lattice Field Theory - Lattice 2011

July 10-16, 2011

Squaw Valley, Lake Tahoe, California

\footnotetext{
${ }^{*}$ Speaker.
} 


\section{Introduction and Motivation}

The low-lying spectrum of the continuum Dirac operator has been described successfully in terms of chiral Lagrangians or Random Matrix Theory [1,2]. The reason for this success is that the smallest eigenvalues are of order $1 / V$, which is deep inside the $\varepsilon$-domain. In this domain the static part of the chiral Lagrangian, which is equivalent to Random Matrix Theory, factorizes from the partition function, and determines properties of the low-lying Dirac eigenvalues. In this lecture we discuss the extension of these results to nonzero lattice spacing. We can distinguish two different types of discretization effects: those that do not affect the symmetries of the Dirac operator and those that do affect its symmetries. In the first case, discretization effects can be absorbed into a redefinition of the low-energy constants. In the second case, the discretization errors take us outside the universality class which requires additional terms in the chiral Lagrangian. The two main discretization schemes are staggered fermions and Wilson fermions and both break the symmetries of the continuum Dirac operator. In this talk we will discuss recent results $[3,4,5,6]$ for the the Wilson Dirac operator including its dependence on the topological index and the number of flavors. The confirmation of these results by lattice simulations $[7,8]$ will be discussed elsewhere in these Proceedings. For recent progress on the staggered Dirac operator see [9].

We will consider discretization effects of the Wilson Dirac operator

$$
D_{W}=\frac{1}{2} \gamma_{\mu}\left(\nabla_{\mu}+\nabla_{\mu}^{*}\right)-\frac{1}{2} a \nabla_{\mu}^{*} \nabla_{\mu} .
$$

Because of its $\gamma_{5}$ Hermiticity it is advantageous to analyze the spectrum of the Hermitian Dirac operator $[10,11,12,13]$

$$
D_{5} \equiv \gamma_{5}\left(D_{W}+m\right)=D_{5}^{\dagger}
$$

Since $D_{W}$ is nonhermitian, its eigenvalues are complex, but because of the $\gamma_{5}$ Hermiticity, they occur in complex conjugate pairs or are real. Therefore the complex eigenvalues can collide with the real axis and turn into a pair of real eigenvalues. This is different from QCD at nonzero chemical potential [14] where the nonzero Dirac eigenvalues are complex and occur in pairs of opposite sign. Then both their real and imaginary parts have to vanish for a pair to collide with the real axis.

In this talk we report on results for discretization effects on spectra of the Wilson Dirac operator of QCD with dynamical quarks. A direct evaluation of the generating function is complicated, but using a diffusion method [15] we obtain compact expressions for correlation functions of Dirac eigenvalues which we here further simplified by exploiting an underlying Pfaffian structure [16].

We start from a chiral Lagrangian, but our results can also be derived from the corresponding chiral Random Matrix Theory [3, 4]. Recently, the joint eigenvalue distribution of both the Wilson Dirac operator [17] and the hermitian Wilson Dirac operator [18] have been obtained from RMT.

\section{Spectrum of the Dirac Operator}

In order to access the spectrum of $D_{5}$, we introduce an axial mass $z$

$$
\operatorname{det}\left(D_{w}+m+\gamma_{5} z\right)=\operatorname{det}\left(\gamma_{5}\left(D_{W}+m\right)+z\right) .
$$




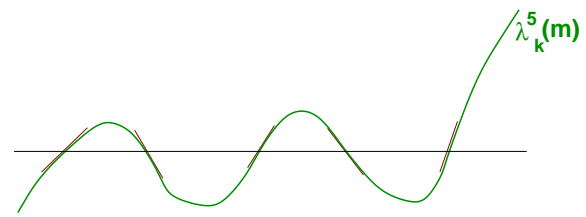

Figure 1: Spectral flow of eigenvalues of $D_{5}$ versus the quark mass $m$.

The low-energy limit of the corresponding partition function is given by a chiral Lagrangian that up to low energy constants is uniquely determined by symmetries [19, 20, 21]

$$
\mathscr{L}(m, z ; a)=\frac{1}{2} m \Sigma \operatorname{Tr}\left(U+U^{\dagger}\right)+\frac{1}{2} z \Sigma \operatorname{Tr}\left(U-U^{\dagger}\right)-a^{2} W_{8} \operatorname{Tr}\left(U^{2}+U^{-2}\right) .
$$

In the microscopic domain, where the combinations $m V, z V$ and $a^{2} V$ are kept fixed in the thermodynamic limit, the $m, z$ and $a$-dependence of the partition function resides in its zero momentum part that factorizes from the nonzero momentum part [3]

$$
Z_{N_{f}}^{V}(m, z ; a)=\int_{U \in U\left(N_{f}\right)} d U \operatorname{det}^{v} U e^{\frac{1}{2} m V \Sigma \operatorname{Tr}\left(U+U^{\dagger}\right)+\frac{1}{2} z V \Sigma \operatorname{Tr}\left(U-U^{\dagger}\right)-a^{2} V W_{8} \operatorname{Tr}\left(U^{2}+U^{-2}\right)} .
$$

To this order the chiral Lagrangian also contains $-a^{2} W_{6}\left[\operatorname{Tr}\left(U+U^{\dagger}\right)\right]^{2}-a^{2} W_{7}\left[\operatorname{Tr}\left(U-U^{\dagger}\right)\right]^{2}$ but they can be added to the mass term after linearizing the squares at the expense of a Gaussian integral and will not be considered below.

Since small Dirac eigenvalues are very sensitive to the index, $v$, of the Dirac operator we will work at fixed $v$. This index is determined from the spectral flow of the eigenvalues of $D_{5}(m)$ $[22,23]$ (see Fig. 1). At the crossing point, $m_{c}$, with the real axis we have (for small $a$, the physical part of the lattice Dirac spectrum can be separated from the unphysical part)

$$
\gamma_{5}\left(D_{W}+m_{c}\right) \phi=0 \quad \Longrightarrow \quad D_{W} \phi=-m_{c} \phi .
$$

Note that multiple crossings may occur. Therefore the number of real eigenvalues of $D_{w}$ is at least equal to its index. The total number of flow lines with a net flow across the real axis is a topological invariant of the Dirac operator. It can be written as a sum over eigenvalues of $D_{5}$ [22]

$$
v=\sum_{m_{k}, \lambda^{5}\left(m_{k}\right)=0} \operatorname{sign}\left(\frac{d \lambda^{5}(m)}{d m}\right)_{m=m_{k}}=\sum_{k} \operatorname{sign}\left(\left\langle\phi_{k}\left|\gamma_{5}\right| \phi_{k}\right\rangle\right) .
$$

The $\theta$-dependence of the QCD partition function is given by

$$
Z_{N_{f}}(m, z ; a, \theta)=\sum_{v} e^{i v \theta} Z_{N_{f}}^{v}(m, z ; a)
$$

In the continuum limit, because of the anomaly, the factor $\exp (i v \theta)$ can be written as the Jacobian of an axial transformation on the quark fields. At nonzero $a$ the natural extension is (See [24].)

$$
m \rightarrow e^{i \theta \gamma_{5} / N_{f}} m, \quad a \rightarrow e^{i \theta \gamma_{5} / N_{f}} a .
$$

As is the case in the continuum limit, the partition function at fixed $v$ is defined by inverting the decomposition (2.6), which also can be applied to the chiral Lagrangian

$$
Z_{N_{f}}^{v}(m, z ; a)=\frac{1}{2 \pi} \int d \theta e^{i v \theta} Z_{N_{f}}(m, z ; a, \theta),
$$



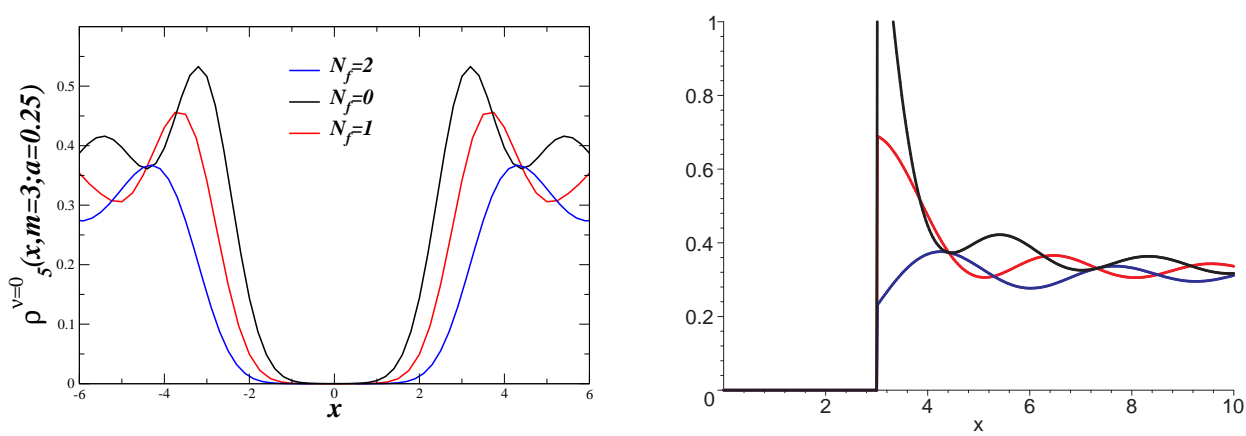

Figure 2: The spectral density of $\gamma_{5}\left(D_{W}+m\right)$ for $m=3, v=0$ and $a=0$ (right) and $a=0.25$ (left) for $N_{f}=0, N_{f}=1 N_{f}=2$.

where the $\theta$-dependence of the chiral Lagrangian is induced by the transformation (2.7).

The generating functions for the Wilson Dirac spectrum are given by [3]

$$
Z_{N_{f}+p \mid p}^{v}\left(m, z_{k}, z_{k}^{\prime} ; a\right)=\left\langle\operatorname{det}^{N_{f}}\left(D_{W}+m\right) \prod_{k=1}^{p} \frac{\operatorname{det}\left(D_{W}+m+\gamma_{5} z_{k}\right)}{\operatorname{det}\left(D_{W}+m+\gamma_{5} z_{k}^{\prime}\right)}\right\rangle_{v} .
$$

The resolvent $(p=1)$ and the spectral density are given by

$$
G_{N_{f}+1 \mid 1}^{v}(z, m ; a)=\left.\lim _{z^{\prime} \rightarrow z} \frac{d}{d z} Z_{N_{f}+1 \mid 1}^{v}\left(m, z, z^{\prime} ; a\right)\right|_{z^{\prime}=z}, \quad \rho_{5}^{v}\left(\lambda^{5}, m ; a\right)=\left.\frac{1}{\pi} \operatorname{Im} G^{v}(z, m ; a)\right|_{z=\lambda^{5}} .
$$

For $z \ll \Lambda_{\mathrm{QCD}}$ the $z$-dependence of the generating function is given by a chiral Lagrangian that is uniquely determined by symmetries that are compatible with the convergence of the bosonic integrals. In the microscopic domain the generating function reduces to the graded integral $[3,2]$

$Z_{N_{f}+1 \mid 1}^{V}(m, z, a)=\frac{1}{Z_{N_{f}}^{V}(m ; a)} \int_{U \in G l\left(N_{f}+1 \mid 1\right)} d U \operatorname{Sdet}^{v}(U) e^{i \frac{1}{2} m V \Sigma \operatorname{Tr}\left(U-U^{\dagger}\right)+i \frac{1}{2} V \Sigma \operatorname{Tr}\left(\zeta U+U^{\dagger} \zeta\right)-i^{2} a^{2} V W_{8} \operatorname{Tr}\left(U^{2}+U^{-2}\right)}$

where, $\zeta_{3}=\operatorname{diag}\left(0, \cdots, 0, z, z^{\prime}\right)$. The transformation $U \rightarrow i U$ is required to get convergent integrals in the noncompact sector for $W_{8}>0$ (for which the Aoki phase [25] can occur).

A direct evaluation of the integrals over the supergroup becomes very laborious for $N_{f} \geq 1$ but they still can be worked out for one flavor $[5,26]$. However, for degenerate quark masses the expressions can be simplified by starting from the identity

$$
e^{a^{2} \operatorname{Trg}\left(U^{2}+U^{\dagger^{2}}\right)}=e^{-2 N_{f} a^{2}+a^{2} \operatorname{Trg}\left(U+U^{\dagger}\right)^{2}}=e^{-2 N_{f} a^{2}} \int d \sigma e^{\operatorname{Trg} \sigma^{2} / 16 a^{2}+\frac{i}{2} \operatorname{Trg} \sigma\left(U+U^{\dagger}\right)},
$$

where $\sigma$ is an $\left(N_{f}+p \mid p\right)$ graded "Hermitian" matrix. In a diagonal representation of $\sigma$ the partition function can be expressed in terms of an integral over shifted quark masses. The result for the resolvent is given by [6]

$$
G_{N_{f}+1 \mid 1}^{v}(m, z ; a)=\frac{1}{Z_{N_{f}}^{v}(m ; a)} \int d S J(S) e^{\left(1 / 16 a^{2}\right) \operatorname{Trg}(S-z)^{2}} \operatorname{Sdet}^{v}(S-m) Z_{N_{f}+1 \mid 1}^{v}\left(\left\{\sqrt{m^{2}-S_{k}^{2}}\right\} ; a=0\right),
$$

where $J(S)$ is the Jacobian of transforming $\sigma$ to the diagonal representation $S$. 

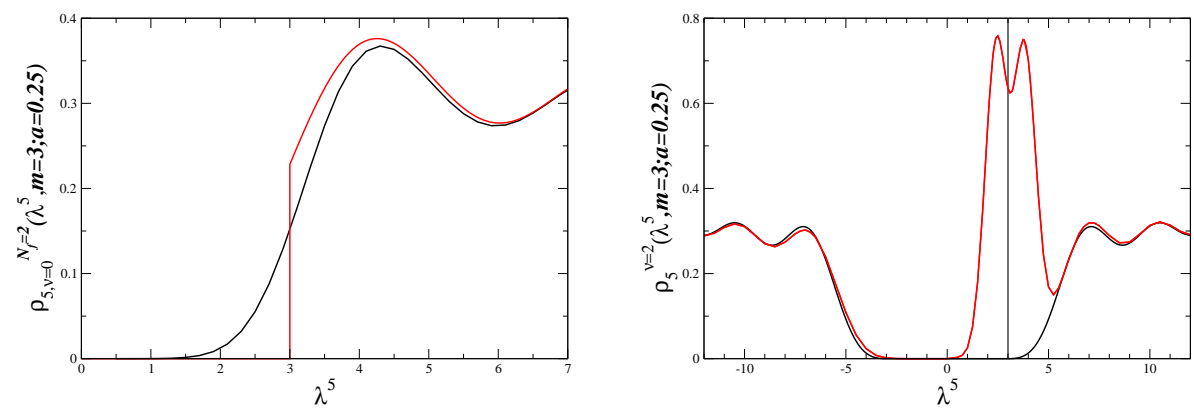

Figure 3: Spectral density for two flavors with quark mass $m V \Sigma=3$ for $v=0$ (left) and $v=2$ (right). We show the result for $a=0$ (black) and $a W_{8} \sqrt{V}=0.25$ (red).

\subsection{Effect of Light Flavors}

For $a=0$ the spectral density of $\gamma_{5}\left(D_{W}+m\right)$ is obtained by a simple variable transformation of the spectral density $\rho^{v}(x)$ of $D_{W}: \rho_{5}^{v}(x)=x / \sqrt{x^{2}-m^{2}} \rho^{v}\left(\sqrt{x^{2}-m^{2}}\right) \theta(|x|-|m|)$ (See Fig. 2 (right)). For $a \neq 0$ the result for the spectral density follows from the resolvent (2.10) (see Fig. 2 (left)). Although, for $N_{f}=1$ and $N_{f}=2$ the integrals in (2.10) can still be evaluated directly, it becomes computationally expensive. However, because of an underlying Pfaffian structure [16], the integrals can be written as the sum of products of two and one dimensional integrals.

The three dimensional integral for one flavor resolvent (2.10) can be rewritten as

$$
G_{2 \mid 1}^{v}(z, m ; a)=G_{1 \mid 1}^{v}(z, z, m ; a)+G_{1 \mid 1}^{v}(0, z, m ; a) \frac{Z_{1}^{v}(z, m ; a)}{Z_{1}^{v}(0, m ; a)}-Z_{2}^{v}(0, z, m ; a) \frac{Z_{0 \mid 1}^{v}(z, m ; a)}{Z_{1}^{v}(0, m ; a)},
$$

where the first term is the quenched resolvent $G_{1 \mid 1}^{v}(z, z, m ; a)$ with

$G_{1 \mid 1}^{v}\left(z_{1}, z_{2}, m ; a\right)=\frac{-1}{16 \pi a^{2}} \int \frac{d s d t}{t-i s} e^{-\left[\left(s+i z_{1}\right)^{2}+\left(t-z_{2}\right)^{2}\right] / 16 a^{2}} \frac{(m-i s)^{v}}{(m-t)^{v}} \tilde{Z}_{1 \mid 1}^{v}\left(\left(m^{2}+s^{2}\right)^{1 / 2},\left(m^{2}-t^{2}\right)^{1 / 2}\right)$.

The various partition functions that enter in the expression are given by

$$
\begin{aligned}
Z_{0 \mid 1}^{v}(z, m ; a)= & \frac{1}{\sqrt{16 \pi a^{2}}} \int d t e^{-(t-z)^{2} / 16 a^{2}} \frac{\left(m^{2}-t^{2}\right)^{v / 2}}{(t-m)^{v}} K_{v}\left(\left(m^{2}-t^{2}\right)^{1 / 2}\right) \\
Z_{1}^{v}(z, m ; a)= & \frac{1}{\sqrt{16 \pi a^{2}}} \int d s e^{-(s+i z)^{2} / 16 a^{2}} \frac{(i s-m)^{v}}{\left(m^{2}+s^{2}\right)^{v / 2}} I_{v}\left(\left(m^{2}+s^{2}\right)^{1 / 2}\right) \\
Z_{2}^{v}\left(z_{1}, z_{2}, m ; a\right)= & \frac{1}{16 \pi a^{2}} \int d s_{1} d s_{2}\left(i s_{1}-i s_{2}\right) e^{\left[\left(i s_{1}-z_{1}\right)^{2}+\left(i s_{2}-z_{2}\right)^{2}\right] / 16 a^{2}}\left(i s_{1}-m\right)^{v}\left(i s_{2}-m\right)^{v} \\
& \times \tilde{Z}_{2}^{v}\left(\left(m^{2}+s_{1}^{2}\right)^{1 / 2},\left(m^{2}+s_{2}^{2}\right)^{1 / 2}\right), \\
\tilde{Z}_{1 \mid 1}^{v}(x, y)= & \frac{y^{v}}{x^{v}}\left[y K_{v+1}(y) I_{v}(x)+x K_{v}(y) I_{v+1}(x)\right], \quad \tilde{Z}_{2}^{v}(x, y)=\frac{y I_{v+1}(y) I_{v}(x)-x I_{v+1}(x) I_{v}(y)}{x^{v} y^{v}\left(y^{2}-x^{2}\right)} .
\end{aligned}
$$

Likewise the four-dimensional integral (2.10) for the $N_{f}=2$ resolvent be re-expressed as

$$
\begin{aligned}
& G_{3 \mid 1}^{v}(z, m ; a) \\
= & G_{1 \mid 1}^{v}(z, z, m ; a)-\left.\frac{i d G_{1 \mid 1}\left(z_{1}, z, m ; a\right)}{d z_{1}}\right|_{z_{1}=0} \frac{Z_{2}^{v}(0, z, m ; a)}{Z_{2}^{v}(m ; a)}+\left.i \frac{G_{1 \mid 1}^{v}(0, z, m ; a)}{Z_{2}^{v}(m ; a)} \frac{d Z_{2}^{v}\left(z_{1}, z, m ; a\right)}{d z_{1}}\right|_{z_{1}=0}
\end{aligned}
$$


where

$$
\begin{aligned}
Z_{2}^{v}(m ; a)= & \frac{i}{\pi\left(16 a^{2}\right)^{2}} \int d s_{1} d s_{2}\left(i s_{1}-i s_{2}\right)^{2} e^{\left[\left(i s_{1}-z_{1}\right)^{2}+\left(i s_{2}-z_{2}\right)^{2}\right] / 16 a^{2}}\left(i s_{1}-m\right)^{v}\left(i s_{2}-m\right)^{v} \\
& \times \tilde{Z}_{2}^{v}\left(\left(m^{2}+s_{1}^{2}\right)^{1 / 2},\left(m^{2}+s_{2}^{2}\right)^{1 / 2}\right) .
\end{aligned}
$$

These simplified expressions for the unquenched resolvents have not been presented previously.

\subsection{Density of Real Eigenvalues}

The Wilson Dirac operator has as least $v$ real eigenvalues in the physical sector of the Dirac spectrum. Because the eigenvalues of the Wilson Dirac operator occur in complex conjugate pairs, additional real eigenvalues may be produced when a pair collides with the real axis. Depending on the expectation value of $\gamma_{5}$ the density of the real eigenvalues may be decomposed as [17]

$$
\rho_{\text {real }}^{v}\left(\lambda^{W}\right)=\rho_{r}^{v}\left(\lambda^{W}\right)+\rho_{l}^{v}\left(\lambda^{W}\right) .
$$

The distribution of the chiralities over real part of the spectrum of $D_{W}$

$$
\rho_{\chi}^{v}\left(\lambda^{W}\right)=\left\langle\sum_{\lambda_{k}^{W} \in \mathbb{R}} \delta\left(\lambda^{W}+\lambda_{k}^{W}\right) \operatorname{sign}\left(\left\langle k\left|\gamma_{5}\right| k\right\rangle\right)\right\rangle_{v}=\rho_{l}^{v}\left(\lambda^{W}\right)-\rho_{r}^{v}\left(\lambda^{W}\right),
$$

was derived from the chiral Lagrangian (2.9) both quenched [3,4] and with dynamical quarks $[5,6]$. We have that

$$
\rho_{\chi}^{v}\left(\lambda^{W}\right) \leq \rho_{\text {real }}^{v}\left(\lambda^{W}\right) \quad \text { and } \quad \int d \lambda^{W} \rho_{\chi}^{v}\left(\lambda^{W}\right)=\left\langle\sum_{\lambda_{k}^{W} \in \mathbb{R}} \operatorname{sign}\left(\left\langle k\left|\gamma_{5}\right| k\right\rangle\right)\right\rangle_{v}=v
$$

The expression for $\rho_{\chi}^{v}\left(\lambda^{W}\right)$ can again be simplified to at most two-dimensional integrals.

The calculation of the density of real eigenvalues is more complicated. However, starting from a random matrix theory for the Wilson Dirac operator, it was possible to derive the joint eigenvalue probability density of $D_{W}$ [17] with the total number of real eigenvalues given by [17,27]

$$
\int d \lambda^{W} \rho_{\text {real }}^{v}\left(\lambda^{W}\right)=v+O\left(\left(V a^{2}\right)^{v+1}\right) .
$$

\section{Conclusions}

We have obtained the lattice spacing dependence of the microscopic spectral density and all spectral correlators of the Hermitian Wilson Dirac operator with degenerate dynamical quark masses. This makes it possible to extract the low-energy constant $W_{8}$ from the distribution of the smallest eigenvalues. It should satisfy the constraint due to $\gamma_{5}$-Hermiticity that $W_{8}>0$ (see also the recent discussion in [28]). These results can be extended to include the two other low-energy constants to this order. Our results also explain the lattice result that the width of the distribution of the smallest eigenvalue scales as $a / \sqrt{V}$. More results can be obtained using a random matrix formulation of the chiral Lagrangian. Then powerful random matrix methods make it possible to obtain all spectral correlation functions of $D_{W}$ in the microscopic domain.

Acknowledgments: This work was supported by U.S. DOE Grant No. DE-FG-88ER40388 (JV) and the Sapere Aude program of The Danish Council for Independent Research (KS). Gernot Akemann, Poul Damgaard, Thomas Guhr and Mario Kieburg are thanked for useful discussions. 


\section{References}

[1] E. V. Shuryak and J. J. M. Verbaarschot, Nucl. Phys. A 560, 306 (1993) [hep-th/9212088];

J. J. M. Verbaarschot and I. Zahed, Phys. Rev. Lett. 70, 3852 (1993) [hep-th/9303012];

J. J. M. Verbaarschot, Phys. Rev. Lett. 72, 2531 (1994) [hep-th/9401059].

[2] P. H. Damgaard, J. C. Osborn, D. Toublan and J. J. M. Verbaarschot, Nucl. Phys. B 547, 305 (1999) [hep-th/9811212].

[3] P. H. Damgaard, K. Splittorff and J. J. M. Verbaarschot, Phys. Rev. Lett. 105, 162002 (2010).

[arXiv:1001.2937 [hep-th]].

[4] G. Akemann, P. H. Damgaard, K. Splittorff, J. J. M. Verbaarschot, Phys. Rev. D 83, 085014 (2011) [arXiv:1012.0752 [hep-lat]].

[5] G. Akemann, P. H. Damgaard, K. Splittorff, J. J. M. Verbaarschot, PoS LATTICE2010, 079 (2010). [arXiv:1011.5121 [hep-lat]].

[6] K. Splittorff, J. J. M. Verbaarschot, Phys. Rev. D84, 065031 (2011). [arXiv:1105.6229 [hep-lat]].

[7] P. H. Damgaard, U. M. Heller, K. Splittorff, [arXiv:1110.2851 [hep-lat]].

[8] A. Deuzeman, U. Wenger, J. Wuilloud, [arXiv:1110.4002 [hep-lat]].

[9] J. C. Osborn, Phys. Rev. D83, 034505 (2011). [arXiv:1012.4837 [hep-lat]].

[10] L. Del Debbio, L. Giusti, M. Lüscher, R. Petronzio and N. Tantalo, JHEP 0602, 011 (2006) [hep-lat/0512021]; JHEP 0702, 056 (2007) [hep-lat/0610059].

[11] M. Golterman, S. R. Sharpe, R. L. Singleton, Jr., Phys. Rev. D71, 094503 (2005). [hep-lat/0501015].

[12] S. R. Sharpe, Phys. Rev. D 74, 014512 (2006) [arXiv:hep-lat/0606002].

[13] S. Necco, A. Shindler, [arXiv:1101.1778 [hep-lat]].

[14] G. Akemann, J. C. Osborn, K. Splittorff, J. J. M. Verbaarschot, Nucl. Phys. B712, 287-324 (2005). [hep-th/0411030].

[15] T. Guhr, Annals Phys. 250, 145-192 (1996).

[16] M. Kieburg, [arXiv:1109.5109 [math-ph]].

[17] M. Kieburg, J. J. M. Verbaarschot, S. Zafeiropoulos, [arXiv:1109.0656 [hep-lat]].

[18] G. Akemann, T. Nagao, JHEP 1110, 060 (2011). [arXiv:1108.3035 [math-ph]].

[19] S. R. Sharpe and R. L. Singleton, Phys. Rev. D 58, 074501 (1998) [hep-lat/9804028].

[20] G. Rupak and N. Shoresh, Phys. Rev. 66, 054503 (2002), [arXiv:hep-lat/0201019].

[21] O. Bär, G. Rupak and N. Shoresh, Phys. Rev. D 70, 034508 (2004), [arXiv:hep-lat/0306021].

[22] S. Itoh, Y. Iwasaki and T. Yoshie, Phys. Rev. D 36, 527 (1987).

[23] K. M. Bitar, U. M. Heller and R. Narayanan, Phys. Lett. B 418, 167 (1998). [arXiv:hep-th/9710052].

[24] S. R. Sharpe, Phys. Rev. D 79, 054503 (2009) [arXiv:0811.0409 [hep-lat]].

[25] S. Aoki, Phys. Rev. D 30 (1984) 2653.

[26] R. N. Larsen, arXiv:1110.5744 [hep-th].

[27] M. Kieburg, J. J. M. Verbaarschot, S. Zafeiropoulos, [arXiv:1110.2690 [hep-lat]].

[28] M. T. Hansen, S. R. Sharpe, [arXiv:1111.2404 [hep-lat]]. 